\title{
Valores de pressão arterial de cães da raça Golden Retriever clinicamente sadios
}

\author{
Blood pressure levels in clinically healthy Golden Retriever
}

\author{
Arine PELLEGRINO ${ }^{1}$; Lilian Caram PETRUS $^{1}$; Fernanda Lie YAMAKI ${ }^{1}$; André Luís Fernandes dos \\ SANTOS $^{1}$; Maria Helena Matiko Akao LARSSON ${ }^{1}$
}

${ }^{1}$ Departamento de Clínica Médica da Faculdade de Medicina Veterinária e Zootecnia da Universidade de São Paulo, São Paulo-SP

\begin{abstract}
Resumo
A hipertensão arterial sistêmica é uma enfermidade que afeta tanto cães quanto gatos e apresenta grande importância na prática da clínica veterinária. É caracterizada pelo aumento sustentado da pressão arterial sistêmica sistólica e/ou diastólica e pode levar a consequências deletérias, principalmente em rins, coração, olhos e sistema nervoso central. Diferentes estudos já foram realizados para a avaliação de técnicas de medida da pressão arterial em cães, comparando diferentes técnicas, com animais em diversos estados de consciência e em diferentes posições. A faixa de normalidade pode variar fisiologicamente nos cães, principalmente de acordo com a raça, sexo, idade, temperamento, condições patológicas, exercícios e dieta. Sabendo-se que o Golden Retriever pode apresentar distrofia muscular canina ligada ao cromossomo X e pode desenvolver cardiomiopatia dilatada e hipertensão sistêmica, no presente estudo, fez-se a mensuração da pressão arterial (por meio de dispositivo de Doppler) em 38 cães clinicamente sadios da referida raça com o propósito de obter parâmetros cardiovasculares que possam servir de referência. A partir dos resultados, pôdese concluir que alterações significativas no peso corpóreo, principalmente dos três aos seis meses de idade, influenciam nos valores de pressão arterial; que a pressão arterial sistólica é maior em machos do que em fêmeas; e que os valores de pressão arterial são variáveis dentro de uma mesma raça canina.
\end{abstract}

Palavras-chave: Pressão arterial sistêmica. Golden Retriever. Hipertensão. Distrofia muscular.

\begin{abstract}
Arterial hypertension is a disease that affects both dogs and cats and presents great importance in veterinary clinic practice. It is characterized by sustained increase in systolic blood pressure systemic and / or diastolic and can cause deleterious consequences, especially in kidney, heart, eyes and central nervous system. Different studies have been conducted for the evaluation of techniques for measuring blood pressure in dogs, comparing different techniques, with animals in various states of consciousness and in different positions. The range of normality can vary physiologically in dogs, mainly according to breed, sex, age, temperament, pathological conditions, exercise and diet. Knowing that the Golden Retriever can submit muscular dystrophy canine linked to chromosome X and can develop dilated cardiomyopathy and systemic hypertension, in this study, the blood pressure (with Doppler) of 38 clinically healthy dogs of that breed was measure with the purpose to obtain parameters that could serve as a reference. From the results, we concluded that significant changes in body weight, mainly from three to six months, influence the blood pressure values, whereas systolic blood pressure is higher in males than in females, and that the pressure values pressure are variables within the same breed of dog.
\end{abstract}

Keywords: Systemic arterial pressure. Golden Retriever. Hypertension. Muscular dystrophy.

\section{Introdução}

Pressão arterial sistêmica é a pressão que o sangue exerce na parede das artérias e nos grandes vasos. A hipertensão arterial é uma enfermidade que afeta tanto cães quanto gatos e apresenta grande importância na prática da clínica veterinária ${ }^{1}$. É caracterizada pelo aumento sustentado da pressão arterial sistêmica sistólica e/ou diastólica e pode levar a consequências deletérias, principalmente em rins, coração, olhos e sistema nervoso central, considerados "órgãos alvo" 2,3 . Em cães e gatos, há uma forte correlação entre injúria ocular e hipertensão arterial $^{2}$, como desenvolvimen-

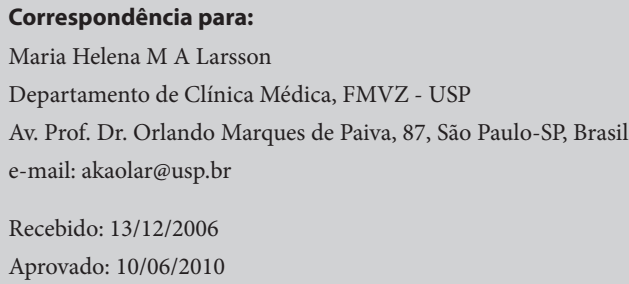


to de retinopatias, coroidopatias, hifema, glaucoma e cegueira. Nos rins, quando a pressão elevada atinge diretamente os capilares glomerulares, há hipertensão glomerular e consequente dano aos glomérulos, com redução progressiva da função renal ${ }^{4}$. Outras manifestações secundárias à hipertensão arterial são hipertrofia do ventrículo esquerdo, disfunção diastólica e insuficiência valvar secundária, devido ao aumento da resistência vascular periférica ${ }^{5}$ e hemorragia cerebral ${ }^{2,3}$.

Na clínica, o diagnóstico de hipertensão sistêmica é baseado na determinação da pressão sanguínea arterial, realizada por métodos diretos (invasivos) ou indiretos (não invasivos). Tilley e Goodwin ${ }^{6}$ classificaram as pressões sanguíneas em quatro grupos diferentes, estabelecendo parâmetros para sua avaliação clínica em: normal (pressão arterial sistólica- PAS entre 110 a $120 \mathrm{mmHg}$ e pressão arterial diastólica- PAD entre 70 a $80 \mathrm{mmHg}$ ); discretamente elevada (PAS entre 120 a $170 \mathrm{mmHg}$ e PAD entre 80 a $100 \mathrm{mmHg}$ ); moderadamente elevada (PAS entre 170 a $200 \mathrm{mmHg}$ e PAD entre 100 a $120 \mathrm{mmHg}$ ) e acentuadamente elevada (PAS acima de $200 \mathrm{mmHg}$ e PAD acima de $120 \mathrm{mmHg}$ ).

Apesar das vantagens dos métodos invasivos em relação à obtenção consistente e precisa dos valores de pressão arterial sistêmica, sendo o padrão para a comparação de métodos indiretos ${ }^{7}$, os mesmos são, no entanto, raramente utilizados na prática, devido às dificuldades da técnica ${ }^{8}$, que incluem sedação ou anestesia do paciente, fatores que, além de pouco práticos, podem reduzir artificialmente a pressão arterial $^{9}$. Por esses motivos, os métodos invasivos de medida da pressão arterial são mais usados em pesquisas ou durante procedimentos anestésicos ${ }^{10}$. Os métodos não invasivos, por outro lado, são preferíveis para a utilização em situações clínicas devido à praticidade de uso e pela possibilidade de ser repetido entre pequenos intervalos de tempo. Porém, as técnicas indiretas são menos precisas quando a pressão sanguínea é baixa, quando há vasoconstrição ou quando ocorre movimentação ex- cessiva do animal ${ }^{7}$. Algumas classes de dispositivos não invasivos para medir pressão sanguínea disponíveis atualmente são o "Doppler" vascular, esfignomanômetro oscilométrico e aparelhos de fotopletismografia ${ }^{1,11}$.

Diferentes estudos já foram realizados para a avaliação de técnicas de medida da pressão arterial em cães, comparando diferentes métodos, com animais em diversos estados de consciência e em diferentes posições ${ }^{12,13}$. Estudos posteriores demonstraram que a faixa de normalidade pode variar fisiologicamente nos cães, principalmente de acordo com a raça e a idade ${ }^{14}$. A pressão sanguínea varia em algumas doenças específicas, sendo normalmente mais elevada em cães com Diabetes mellitus ${ }^{14}$, hiperadrenocorticis$\mathrm{mo}^{15}$, nefropatias e algumas cardiopatias ${ }^{16}$. De acordo com Jepson et al. ${ }^{1}$, a pressão arterial sistólica pode ser aferida em $100 \%$ das tentativas de uso do "Doppler" em gatos conscientes. Em contrapartida, a pressão arterial diastólica pôde ser aferida em apenas $51,4 \%$ das tentativas. Além disso, as médias de pressão arterial diastólica obtidas por dois examinadores, nesse caso, foram estatisticamente diferentes entre si, o que implica que a pressão arterial diastólica obtida por meio do "Doppler" pode não ser confiável. De acordo com Bodey e Michell ${ }^{17}$, a mensuração não invasiva e indireta da pressão arterial é confiável e pode ser rotineiramente utilizada em cães e gatos. Valores de pressão arterial sistólica são bastante variáveis e são influenciados pela raça, idade, sexo, temperamento, condições patológicas, exercícios e pela dieta.

A distrofia muscular canina ligada ao cromossomo $\mathrm{X}$ é reconhecida e melhor caracterizada em cães da raça Golden Retriever, nos quais ocorre de forma espontânea ${ }^{18,19,20,21}$. No homem, alterações cardíacas são complicações reconhecidas em portadores adultos de distrofinopatias ligadas ao cromossomo $\mathrm{X}^{22,23}$. O miocárdio deficiente em distrofina é mais vulnerável à sobrecarga de pressão e os pacientes com DMD podem desenvolver cardiomiopatia dilatada e hipertensão arterial2 $^{24,25,26}$. A distrofia muscular de Duchenne (DMD) 
é uma alteração neuromuscular hereditária comum em humanos; afeta, aproximadamente, um em cada 3.500 indivíduos do sexo masculino com herança ligada ao cromossomo X e é caracterizada por contínua necrose muscular e degeneração, com eventual fibrose e infiltração por tecido adiposo ${ }^{27,28,29,30}$. Camundongos e cães com distrofia muscular, ambos com defeito na distrofina dos tecidos musculares, são modelos para a DMD em humanos ${ }^{31,32,33}$.

O Golden Retriever tem-se tornado bastante comum no Brasil nos últimos anos, apresentando-se como uma raça em ascensão. Devido ao aumento de cruzamentos entre animais de linhagens diversas e sabendo-se da possibilidade de ocorrência de mutações espontâneas que podem levar à distrofia muscular, é de fundamental importância o conhecimento de parâmetros cardiovasculares normais para a referida raça. Portanto, a finalidade do presente estudo é a obtenção de parâmetros de pressão arterial de cães sadios da raça Golden Retriever, para que possam servir de referência.

\section{Material e Método}

Foram utilizados 38 cães sadios da raça Golden Retriever, provenientes do canil de Golden Retriever com distrofia muscular da Faculdade de Medicina Veterinária e Zootecnia da Universidade de São Paulo (GRMD-Brasil), de canis particulares e de proprietários particulares. Todos os animais selecionados foram submetidos a exames físico, bioquímico (avaliação renal, hepática e determinação de eletrólitos) e hematológico para que pudessem ser incluídos no projeto. Os cães procedentes do canil GRMD-Brasil, logo ao nascimento, foram submetidos à avaliação genética e identificados como "sadios" quanto à distrofia muscular por meio do "kit" GFX Genomic Blood DNA Purification, em exame realizado no Centro de Estudos do Genoma Humano do Instituto de Biociências da Universidade de São Paulo.
Os animais do estudo em questão foram distribuídos em dois grupos:

grupo I: animais de até 12 meses de idade (total de 20 cães);

grupo II: animais com idade entre 12 e 36 meses (total de 18 cães).

Devido à grande variação de peso e porte entre os animais do grupo I, composto por filhotes em fase de desenvolvimento, fez-se necessária a subdivisão desta categoria em:

- grupo I-1: animais de até três meses de idade - 0 - 3 (cinco cães);

- grupo I-2: animais entre três e seis meses de idade - 3 - 6 (cinco cães);

- grupo I-3: animais entre seis e nove meses de idade - 6 -9 (cinco cães);

- grupo I-4: animais entre nove e 12 meses de idade - 9 ト 12 (cinco cães).

A determinação da pressão arterial sistólica (PAS), diastólica (PAD) e média (PAM) foi realizada por método não invasivo por meio do dispositivo de "Doppler”, aparelho da marca Medmega ${ }^{\circledR}$, modelo DV610. Foram realizadas, no mínimo, três determinações, considerando-se a média dos valores obtidos; e os valores muito altos e/ou muito baixos foram excluídos da contagem final. Neste procedimento, os animais foram posicionados em decúbito lateral direito, mensurando-se a circunferência do membro torácico do animal, e colocando-se um manguito (com largura correspondente a $40 \%$ da circunferência do membro) ao nível do terço médio do rádio-ulna. $\mathrm{O}$ transdutor foi posicionado entre os coxins do carpo e metacarpo, sobre a região da artéria medial, iniciando-se a insuflação do manguito até a interrupção do pulso. A pressão arterial sistólica (PAS) foi caracterizada no momento do retorno da percepção do pulso (ao desinflar o manguito), a pressão arterial diastólica (PAD) pela modificação no timbre e no som emitido, e a média (PAM) de acordo com a metodologia já estabelecida $^{34,35}$. 
Os valores de pressão arterial foram apresentados na forma de media e desvio padrão e posteriormente analisados por meio do programa "GraphPad Instat"teste de Bonferroni.

\section{Resultados}

Dos 38 cães sadios da raça Golden Retriever avaliados (20 do grupo I e 18 do grupo II), nove eram machos de até 12 meses de idade (45\%), 11 eram fêmeas de até 12 meses de idade (55\%), dez eram machos entre 12 e 36 meses de idade (55\%) e oito eram fêmeas entre 12 e 36 meses de idade (45\%); e os valores e parâmetros obtidos em relação a peso, idade e pressão arterial estão descritos na tabela 1.
Os valores de pressão arterial de cães da raça Golden Retriever de até 12 meses de idade (média de peso corpóreo de 16,2 $\pm 10,998 \mathrm{~kg}$ ) obtidos foram: PAS de $132,196 \pm 14,707 \mathrm{~mm}$ de $\mathrm{Hg}$ X PAD de 83,408 $\pm 8,169$ mm de $\mathrm{Hg}$ e PAM de 99,670 \pm 10,758 mm de Hg. Os valores de pressão arterial de cães da raça Golden Retriever de até 36 meses de idade (média de peso corpóreo de 28,943 $\pm 4,832 \mathrm{~kg}$ ) foram: PAS de 155,235 \pm 22,262 mm de Hg X PAD de 99,801 $\pm 15,155 \mathrm{~mm}$ de $\mathrm{Hg}$ e PAM de $118,279 \pm 18,024 \mathrm{~mm}$ de $\mathrm{Hg}$.

\section{Discussão}

De acordo com Tilley e Goodwin ${ }^{6}$, a pressão arterial é classificada em: normal (PAS entre 110 a 120

Tabela 1- Médias e desvios padrão de peso, idade e parâmetros de pressão arterial de cães da raça Golden Retriever - São Paulo - 2008

\begin{tabular}{cccccc}
\hline Grupos & $\begin{array}{c}\text { Peso } \\
(\mathbf{K g})\end{array}$ & Idade (meses) & $\begin{array}{c}\text { PAS } \\
(\mathbf{m m ~ H g})\end{array}$ & $\begin{array}{c}\text { PAD } \\
(\mathbf{m m ~ H g})\end{array}$ & $\begin{array}{c}\text { PAM } \\
(\mathbf{m m ~ H g})\end{array}$ \\
\hline $\mathbf{I}-\mathbf{1}$ & $2,340^{\mathrm{a}}$ & $1,000^{\mathrm{a}}$ & $115,200^{\mathrm{a}}$ & $76,000^{\mathrm{a}}$ & $89,067^{\mathrm{a}}$ \\
$(\mathbf{n}=\mathbf{5})$ & $\pm 0,26$ & \pm 0 & $\pm 10,034$ & $\pm 9,617$ & $\pm 9,620$ \\
$\mathbf{I - 2}$ & $10,760^{\mathrm{b}}$ & $3,429^{\mathrm{a}}$ & $115,600^{\mathrm{a}}$ & $71,800^{\mathrm{a}}$ & $86,400^{\mathrm{a}}$ \\
$(\mathbf{n}=\mathbf{5})$ & $\pm 5,485$ & $\pm 0,787$ & $\pm 13,446$ & $\pm 5,495$ & $\pm 9,203$ \\
$\mathbf{I} \mathbf{- 3}$ & $23,800^{\mathrm{c}}$ & $6,500^{\mathrm{a}}$ & $145,982^{\mathrm{a}}$ & $93,166^{\mathrm{a}}$ & $110,771^{\mathrm{a}}$ \\
$(\mathbf{n}=\mathbf{5})$ & $\pm 1,351$ & $\pm 0,548$ & $\pm 23,496$ & $\pm 13,162$ & $\pm 17,405$ \\
$\mathbf{I}-\mathbf{4}$ & $27,975^{\mathrm{c}}$ & $10,75^{\mathrm{b}}$ & $152,000^{\mathrm{a}}$ & $92,666^{\mathrm{a}}$ & $112,444^{\mathrm{a}}$ \\
$(\mathbf{n}=\mathbf{5})$ & $\pm 5,898$ & $\pm 0,5$ & $\pm 11,853$ & $\pm 4,403$ & $\pm 6,806$ \\
\hline $\mathbf{I}$ & $16,218^{\mathrm{b}}$ & $5,419^{\mathrm{a}}$ & $132,195^{\mathrm{a}}$ & $83,408^{\mathrm{a}}$ & $99,670^{\mathrm{a}}$ \\
$(\mathbf{n}=\mathbf{2 0})$ & $\pm 10,998$ & $\pm 0,458$ & $\pm 14,707$ & $\pm 8,169$ & $\pm 10,758$ \\
II & $28,943^{\mathrm{c}}$ & $20,800^{\mathrm{c}}$ & $155,235^{\mathrm{b}}$ & $99,801^{\mathrm{b}}$ & $118,279^{\mathrm{b}}$ \\
$(\mathbf{n}=\mathbf{1 8})$ & $\pm 4,832$ & $\pm 8,697$ & $\pm 22,262$ & $\pm 15,155$ & $\pm 18,024$ \\
\hline
\end{tabular}

$\mathbf{n}=$ número de animais; $\mathbf{a}, \mathbf{b}, \mathbf{c}=$ diferenças estatísticas, $\mathbf{P A S}=$ pressão arterial sistólica; $\mathbf{P A M}=$ pressão arterial média; $\mathbf{P A D}$ = pressão arterial diastólica; I-1 = subgrupo I-1 ( 0 a 3 meses de idade); I-2 = subgrupo I-2 ( 3 a 6 meses de idade); I-3 = subgrupo I-3 (6 a 9 meses de idade); I-4 = subgrupo I-4 ( 9 a 12 meses de idade); I = grupo I ( 0 a 12 meses de idade); II = grupo II (12 a 36 meses de idade)

$\mathrm{mmHg}$ e PAD entre 70 a $80 \mathrm{mmHg}$ ); discretamente elevada (PAS entre 120 a $170 \mathrm{mmHg}$ e PAD entre 80 a $100 \mathrm{mmHg}$ ); moderadamente elevada (PAS entre 170 a $200 \mathrm{mmHg}$ e PAD entre 100 a $120 \mathrm{mmHg}$ ) e acentuadamente elevada (PAS acima de $200 \mathrm{mmHg}$ e PAD acima de $120 \mathrm{mmHg}$ ). Porém, o valor de pressão arterial que representa um "aumento anormal" é ainda desconhecido. Muitos autores consideram cães com pressão arterial normal, sem anestesia e sem agitação física, não ultrapassando $160 \mathrm{~mm}$ de $\mathrm{Hg}$ por $100 \mathrm{~mm}$ de Hg; e $180 \mathrm{~mm}$ de $\mathrm{Hg}$ quando estressados ou ansiosos. Pressões sistólicas de 180-200 mm de Hg por 110 $\mathrm{mm}$ de $\mathrm{Hg}$ de diastólica são consideradas no limite ou aumentadas $^{36}$.

Os animais dos subgrupos I-1 e I-2 apresentaram valores de pressão arterial (PAS, PAD e PAM) dentro dos parâmetros normais. Os cães dos subgrupos I-3 e I-4 apresentaram valores de pressão arterial discreta- 
mente elevados. E tanto no grupo I (considerando a média de todos os subgrupos) quanto no grupo II, os valores de pressão arterial obtidos foram classificados como discretamente aumentados (Tabela 1), em relação à classificação de Tilley e Goodwin .

Grande variabilidade foi observada nos valores de pressão arterial entre os animais dos diferentes grupos. Esta variabilidade de resultados pode ser associada com o temperamento e agitação do animal durante o exame, o que pode superestimar os valores. De acordo com o estudo de Kallet, Cowgill e $\mathrm{Kass}^{37}$, valores de frequência cardíaca e de pressão arterial obtidos em ambiente de clínica veterinária são maiores que os obtidos no ambiente familiar do cão. No entanto, para auxiliar na interpretação dos resultados, valores de pressão arterial precisam ser padronizados considerando as interferências do estresse e do ambiente ${ }^{38,39}$.

No presente estudo, pôde-se verificar que a pressão arterial aumentou de acordo com a idade e porte dos animais, ao comparar cães de diferentes subgrupos e grupos (Tabela 1). De acordo com o estudo de Bright e Dentino ${ }^{40}$ sobre padronização de valores de pressão arterial em cães da raça Irish Wolfhounds, verificou-se que a pressão arterial sofre variação de acordo com a idade, sexo, temperamento do animal e posicionamento durante a mensuração. A pressão arterial tende a ser maior nos animais mais velhos $\mathrm{e}$ nos mais ansiosos.

De acordo com Ware ${ }^{36}$, em um estudo com cães normais, a média de pressões encontradas foi de 150 $\mathrm{mm}$ de $\mathrm{Hg}$ por $86 \mathrm{~mm}$ de $\mathrm{Hg}$; e apenas casos com pressão maior que $202 \mathrm{~mm}$ de $\mathrm{Hg}$ por $116 \mathrm{~mm}$ de $\mathrm{Hg}$ foram considerados hipertensão. No presente estudo, de acordo com a classificação de Tilley e Goodwin ${ }^{6}$, um animal do subgrupo I-2, três do subgrupo I-3, cinco do I-4 e oito do grupo II apresentaram valores de pressão arterial discretamente aumentados; um cão do subgrupo I-3 e seis do grupo II apresentaram valores moderadamente aumentados; e todos os ou- tros animais apresentaram valores dentro dos parâmetros de normalidade.

De acordo com Fox, Sisson e Moise ${ }^{41}$ e Ware ${ }^{36}$, diferenças na pressão arterial são observadas com a idade, raça, sexo e outros fatores em animais sadios, bem como nos doentes. Em cães adultos normais, as PAS, PAD e PAM aumentam no final da meia idade, independentemente da definição racial. Machos inteiros apresentam valores superiores aos dos machos castrados; e as fêmeas castradas apresentam os menores valores pressóricos. No presente estudo, todos os animais com aumento moderado da pressão arterial (PAS entre 170-200 mm de Hg e PAD entre 100-120 $\mathrm{mm}$ de $\mathrm{Hg}$ ) eram machos inteiros, sendo a maioria composta por adultos (85,7\%). Os valores mais baixos de pressão arterial (100 mm de $\mathrm{Hg}$ por $60 \mathrm{~mm}$ de $\mathrm{Hg}$, no subgrupo I-1; e $100 \mathrm{~mm}$ de $\mathrm{Hg}$ por $63 \mathrm{~mm}$ de Hg, no subgrupo I-2) foram encontrados em uma fêmea (de um mês de idade) e em um macho (de cinco meses de idade), respectivamente.

Diferenças estatísticas nos valores de pressão arterial sistólica, diastólica e média foram observadas entre os grupos I e II ( $\mathrm{p}<0,001)$, com valores maiores nos animais adultos (Tabela 1). No entanto, não foram encontradas diferenças estatísticas significativas entre os valores de pressão arterial sistólica, diastólica e média entre os diferentes subgrupos do grupo I ( $p>0,05)$. Pôde-se verificar que a PAS nos machos foi maior que nas fêmeas tanto no grupo I quanto no grupo II e na maioria dos subgrupos (Figura 1). Estatisticamente, puderam-se verificar diferenças significativas da PAS entre machos e fêmeas (umpaired t test; $\mathrm{p}$ value $=0,0432$ ), com predomínio de valores maiores nos machos. Pôde-se observar certa tendência de aumento nos valores de pressão arterial sistólica de acordo com o peso e porte dos animais (Figura 2). No entanto, estatisticamente, não foram observadas variações significativas correlacionando pressão arterial e o peso dos animais (umpaired t test). 


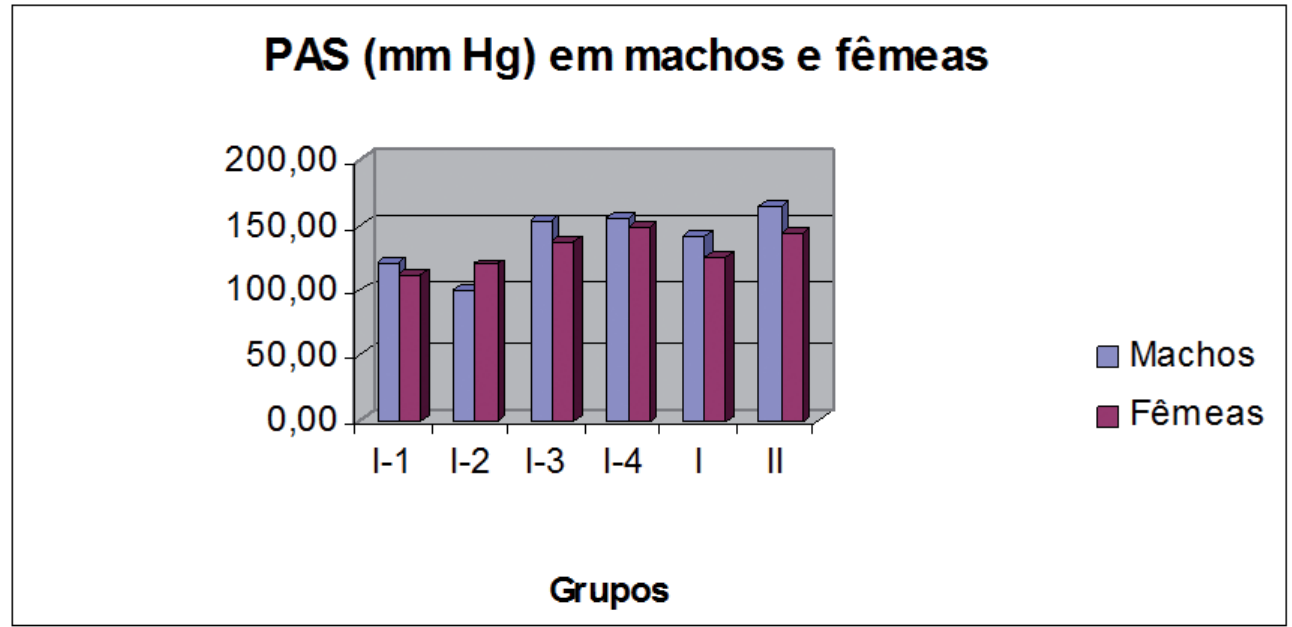

PAS = pressão arterial sistólica $(\mathrm{mm} \mathrm{Hg}) ; \mathbf{I}-1$ = subgrupo I-1 (0 a 3 meses de idade); I-2 = subgrupo I-2 (3 a 6 meses de idade); I-3 = subgrupo I-3 (6 a 9 meses de idade); I-4 = subgrupo I-4 (9 a 12 meses de idade); I = grupo I ( 0 a 12 meses de idade); $\mathbf{I}$ = grupo II (12 a 36 meses de idade).

Figura 1- Média da pressão arterial sistólica - PAS (mm Hg) em machos e fêmeas de diferentes subgrupos e grupos - São Paulo -2008

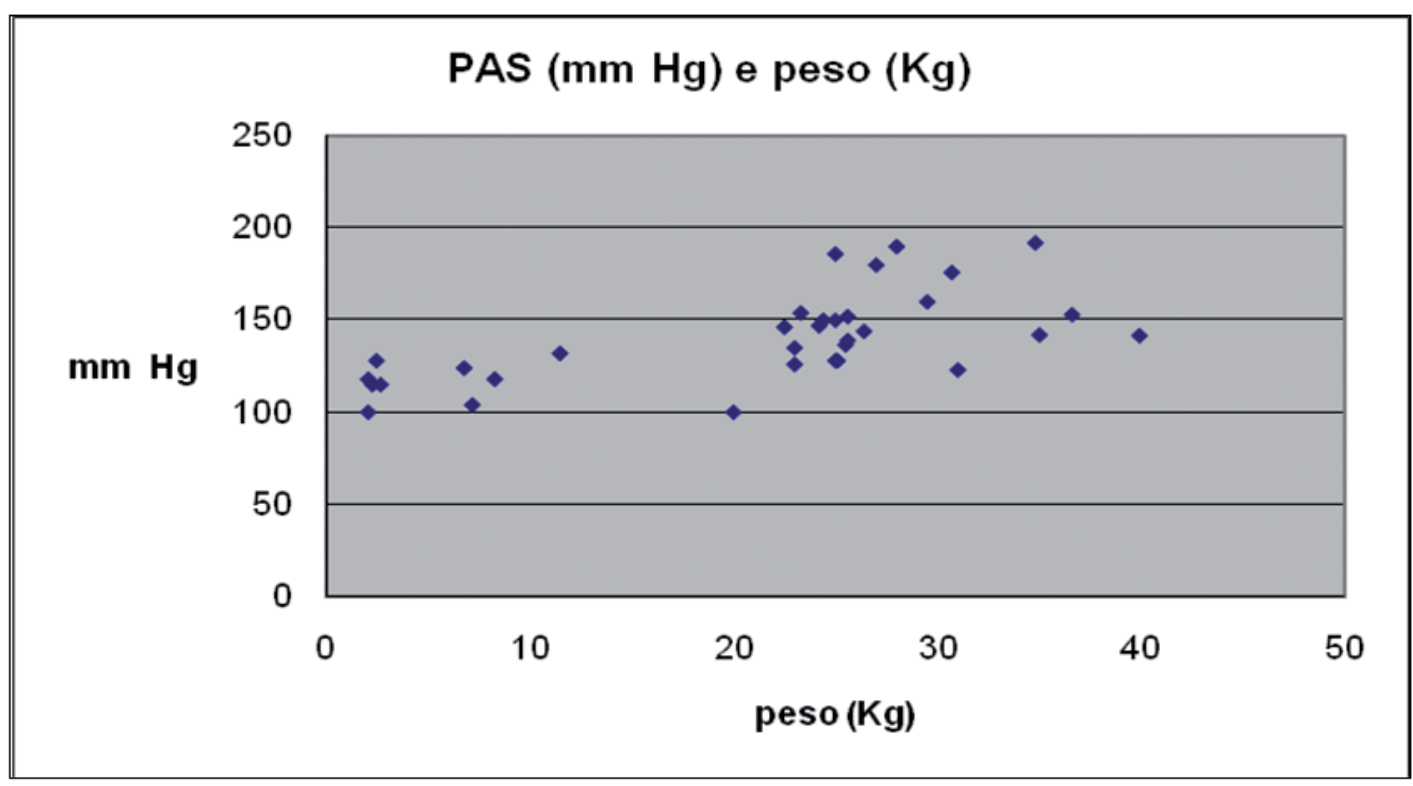

Figura 2 - Variação da pressão arterial sistólica - PAS (mm Hg) em relação ao peso corpóreo (Kg) de cães da raça Golden Retriever - São Paulo - 2008

\section{Conclusões}

De acordo com a análise dos resultados obtidos e com a metodologia utilizada no presente estudo, as seguintes afirmações são possíveis: em cães da raça Golden Retriever, alterações significativas no peso corpóreo, principalmente dos três aos seis meses, influenciam nos valores de pressão arterial; a pressão arterial sistólica é maior em machos do que em fêmeas; os valores obtidos de pressão arterial de cães da raça Golden Retriever de até 12 meses de idade (média de 
peso corpóreo de 16,2 $\pm 10,998 \mathrm{~kg}$ ) foram: PAS de $132,196 \pm 14,707 \mathrm{~mm}$ de $\mathrm{Hg}$ X PAD de 83,408 \pm $8,169 \mathrm{~mm}$ de $\mathrm{Hg}$ e PAM de 99,670 $\pm 10,758 \mathrm{~mm}$ de Hg; os valores obtidos de pressão arterial de cães da raça Golden Retriever de até 36 meses de idade (média de peso corpóreo de 28,943 \pm 4,832 kg)

\section{Referências}

1. JEPSON, R. E.; HARTLEY, V.; MENDL, M.; CANEY, S. M.; GOULD, D. J. A comparison of CAT Doppler and oscillometric memoprint machines for non-invasive blood pressure measurement in conscious cats. Journal of Feline Medicine and Surgery, v. 7, p. 147-152, 2005.

2. BROWN, S. A.; HENIK, R. A. Diagnosis and treatment of systemic hypertension. Veterinary Clinics of North America: Small Animal Practice, v. 28, n. 6, p. 1481-1493, 1998.

3. BROWN, S.; ATKINS, C.; BAGLEY, R.; CARR, A.; COWGILL, L.; DAVIDSON, M.; EGNER, B.; ELLIOTT, J.; HENIK, R.; LABATO, M.; LITTMAN, M.; POLZIN, D.; ROSS, L.; SNYDER, P.; STEPIEN, R. Guidelines for the identification, evaluation, and management of systemic hypertension in dogs and cats. Journal of Veterinary Internal Medicine, v. 21, n. 3, p. 542-558, 2007.

4. BROWN, S. A.; FINCO, D. R.; NAVAR, L. G. Impaired renal autoregulatory ability in dogs with reduced renal mass. Journal of American Society of Nephrology, v. 5, n. 10, p. 1768-1774, 1995.

5. DOUGLAS, P. S.; TALLANT, B. Hypertrophy, fibrosis and diastolic dysfunction in early canine experimental hypertension. Journal of American College of Cardiology, v. 17, n. 2, p. 530, 1991.

6. TILLEY, L. P.; GOODWIN, J. K. Manual de cardiologia para cães e gatos. 3. ed. São Paulo: Roca, 2002. 489 p.

7. PODELL, M. Use of blood pressure monitors. In: BONAGURA, J. D. Kirk's current veterinary therapy XI: small animal practice. Philadelphia: W. B. Saunders, 1992. p. 834-837.

8. PORCIELLO, F.; BIRETTONI, F.; CONTI, M. B.; MARINETTI, C.; ANTOGNONI, M. T.; FRUGANTI, G. Blood pressure measurements in dogs and horses using the oscillometric technique: personal observations. Veterinary Research Communications, v. 28, n. 8, p. 367-369, 2004. Supplement, 1.

9. KITTLESON, M. D.; OLIVER, N. B. Measurement of systemic arterial blood pressure. Veterinary Clinics of North America: Small Animal Practice, v. 13, p. 321-336, 1983.

10.CHALIFOUX, A.; DALLAIRE, A.; BLAIS, D.; LARIVIĖRE, N.; PELLETIER, N. Evaluation of the arterial blood pressure of dogs by two noninvasive methods. Canadian Journal of Comparative Medicine, v. 49, p. 419-423, 1985.

11.STEPIEN, R. L.; RAPOPORT, G. S.; HENIK, R. A.; WENHOLZ, L.; THOMAS, C. B. Comparative diagnostic test characteristics of oscilometric and Doppler ultrasonographic methods in detection of systolic hypertension in dogs. Journal of Veterinary Internal Medicine, v. 17, n. 1, p. 65-72, 2003.

12.STEPIEN, R. L.; RAPOPORT, G. S. Clinical comparison of three methods to measure blood pressure in nonsedated dogs. Journal of the American Veterinary Medical Association, v. 215, n. 11, p. 1623-1628, 1999. foram: PAS de 155,235 $\pm 22,262 \mathrm{~mm}$ de $\mathrm{Hg} \mathrm{X}$ PAD de 99,801 $\pm 15,155 \mathrm{~mm}$ de $\mathrm{Hg}$ e PAM de 118,279 \pm 18,024 mm de Hg; os valores de pressão arterial são variáveis dentro de uma mesma raça canina, tornando imprescindível minuciosa avaliação clínica concomitante.

13.BODEY, A. R.; MICHELLK, A. R.;BOVEE, C.; BURANAKURL C.; GARG, T. Comparison of direct and indirect (oscillometric) measurement of arterial pressure in conscious dogs. Research in Veterinary Science, v. 61, n. 1, p. 17-21, 1996.

14.BODEY, A. R.; MICHELL, A. R. Longitudinal studies of reproducibility and variability of indirect (oscillometric) blood pressure measurements in dogs: evidence for tracking. Research in Veterinary Science, v. 63, n. 1, p. 15-21, 1997.

15.MARCO, V. D.; LARSSON, C. E. Hipertensão secundária ao hiperadrenocorticismo hipofisário canino. Revista Brasileira de Ciência Veterinária, v. 7, p. 80, 2000. Suplemento. Trabalho apresentado no XXI Congresso Brasileiro de Clínicos Veterinários de Pequenos Animais, 2000.

16.LARSSON, M. H. M. A.; D'URSO, F. Determination of arterial pressure by indirect oscillometric method in healthy, cardiac and nephropathic dogs. In: WORLD CONGRESS OF SMALL ANIMAL VETERINARY ASSOCIATION, 23., 1998. Buenos Aires. Anais... Buenos Aires: WSAVA, 1998. v. 2, p. 760-760.

17.BODEY, A. R.; MICHELL, A. R. Epidemiological study of blood pressure in domestic dogs. Journal of Small Animal Practice, v. 37, n. 3, p. 116-125, 1996.

18. VALENTINE, B. A.; WINAND, N. J.; PRADHAN, D.; MOISE, N. S.; DE LAHUNTA, A.; KORNEGAY, J. N.; COOPER, B. J. Canine X-linked muscular dystrophy as an animal model of Duchenne muscular dystrophy: a review. American Journal of Medicine and Genetics, v. 42, n. 3, p. 352-356, 1992.

19.JAZEDJE, T.; DANI, S. U.; ZATZ, M. Establishment of a muscular dystrophy dog colony in Brazil. Genetics and Molecular Biology, v. 23, n. 3, p. 75, 2000. Supplement.

20. SHIMATSO, Y.; KATAGIRI, K.; FURUTA, T.; NAKURA, M.; TANIOKA, Y.; YUASA, K.; TOMOHIRO, M.; KORNEGAY, J. N.; NONAKA, I.; TAKEDA, S. Canine X-linked muscular dystrophy in Japan (CXMDj). Experimental Animal, v. 52, n. 2, p. 93-97, 2003.

21.VAINZOF, M.; AYUB-GUERRIERI, D.; ONOFRE, P. C.; MARTINS, P. C.; LOPES, V. F.; ZILBERZTAJN, D.; MAIA, L. S.; SELL, K.; YAMAMOTO, L. U. Animal models for genetic neuromuscular diseases. Journal of Molecular Neuroscience, v. 34 , n. 3, p. 241-248, 2008.

22.HOOGERWAARD, E. M.; VAN DER WOUW, P. A.; WILDE, A. A.; BAKKER, E.; IPPEL, P. F.; OOSTERWIJK, J. C.; MAJOOR-KRAKAUER, D. F.; VAN ESSEN, A. J.; LESCHOT, N. J.; DE VISSER, M. Cardiac involvement in carriers of Duchenne and Becker muscular dystrophy. Neuromuscular Disorders, v. 9, n. 5, p. 347-351, 1999.

23. NOLAN, M. A.; JONES, O. D.; PEDERSEN, R. L.; JOHNSTON, H. M. Cardiac assessment in childhood carriers of Duchenne and Becker muscular dystrophies. Neuromuscular Disorders, v. 13, n. 2, p. 129-132, 2003. 
24.HUNSAKER, R. H.; FULKERSON, P. K.; BARRY, F. J.; LEWIS, R. P.; LEIER, C. V.; UNVERFERTH, D. V. Cardiac function in Duchennes's muscular dystrophy. The American Journal of Medicine, v. 73, n. 2, p. 235-238, 1982.

25.KAMOGAWA, Y.; BIRO, S.; MAEDA, M.; SETOGUCHI, M.; HIRAKAWA, T.; YOSHIDA, H.; TEI, C. Dystrophyndeficient myocardium is vulnerable to pressure overload in vivo. Cardiovascular Research, v. 50, n. 3, p. 509-515, 2001

26. YUGETA, N.; URASAWA, N.; FUJII, Y.; YOSHIMURA, M.; YUASA, K.; WADA, M. R.; NAKURA, M.; SHIMATSU, Y.; TOMOHIRO, M.; TAKAHASHI, A.; MACHIDA, N.; WAKAO, Y.; NAKAMURA, A.; TAKEDA, S. Cardiac involvement in Beagle-based canine X-linked muscular dystrophy in Japan (CXMDJ): electrocardiography, echocardiographic and morphologic studies. BMC Cardiovascular Disorders, v. 6, p. 47, 2006.

27.MOISE, N. S.; VALENTINE, B. A.; BROWN, C. A.; ERB, H. N.;BECK, K. A.; COOPER, B. J.; GILMOUR, R. F. Duchenne's cardiomyopathy in a canine model: electrocardiographic and echocardiographic studies. Journal of American College of Cardiology, v. 17, n. 4, p. 812-820, 1991.

28.HOWELL, J. M.; FLETCHER,S.; KAKULAS, B. A.; O'HARA, M.; LOCHMULLER, H.; KARPATI, G. Use of the dog model for Duchenne muscular dystrophy in gene therapy trials. Neuromuscular Disorders, v. 7, n. 5, p. 325-328, 1997.

29. CHILDERS, M. K.; OKAMURA, C. S.; BOGAN, D. J.; BOGAN, J. R.; PETROSKI, G. F.; MCDONALD, K.; KORNEGAY, J. N. Eccentric contraction injury in dystrophic canine muscle. Archives of Physical Medicine Rehabilitation, v. 83, n. 11, p. 1572-1578, 2002.

30.HAINSEY, T. A.; SENAPATI, S.; KUHN, D. E.; RAFAEL, J. A. Cardiomyopathic features associated with muscular dystrophy are independent of dystrophin absence in cardiovasculature. Neuromuscular Disorders, v. 13, n. 4, p. 294-302, 2003.

31.NGUYEN, F.; CHEREL, Y.; GUIGAND, L.; GOUBAULTLEROUX, I.; WYERS, M. Muscle lesions associated with dystrophin deficiency in neonatal Golden Retriever puppies. Journal of Comparative Pathology, v. 126, n. 2-3, p. 100$108,2002$.
32.BOGDANOVICH, S.; PERKINS, K. J.; KRAG. T. O.; KHURANA, T. S. Therapeutics for Duchenne muscular dystrophy: current approaches and futures directions. Journal of Molecular Medicine, v. 82, n. 2, p. 102-115, 2004.

33.COLLINS, C. A.; MORGAN, J. E. Duchenne's muscular dystrophy: animal models used to investigate pathogenesis and develop therapeutic strategies. International Journal of Experimental Pathology, v. 84, n. 4, p. 165-172, 2003.

34.BROWN, S. A.; HENIK, R. A.; FINCO, D. R. Diagnosis of systemic hypertension in dogs and cats. In: BONAGURA, J. D. Kirk's current veterinary therapy-Small animal practice. 13. ed. Philadelphia: W.B. Saunders, 2000. p. 835-838.

35. WARE, W. Cardiovascular disease in small animal medicine. 1. ed. London: Manson Publishing Ltd, 2007. 396 p.

36. WARE, W. A. Hipertensão arterial sistêmica. In: NELSON, R. W.; COUTO, C. G. Medicina interna de pequenos animais. 3. ed. Rio de Janeiro: Guanabara Koogan, 2005. p. 150-159.

37.KALLET, A. J.; COWGILL, L. D.; KASS, P. H. Comparison of blood pressure measurements obtained in dog by use of indirect oscillometry in a veterinary clinic versus at home. Journal of American Veterinary Association, v. 210, n. 5, p. 651-654, 1997.

38. MISHIMA, M.; WATANABE, T.; FUIII, K.; MAEDA, H.; WAKAO, Y.; TAKAHASHI, M. A. A clinical evaluation of blood pressure through non-invasive measument using the oscillometric procedure in conscious dogs. Journal of Veterinary Medicine Science, v. 59, n. 11, p. 989-993, 1997.

39. REMILLARD, R.L. et al. Variance of indirect blood pressure measurements and prevalence of hypertension in clinically normal dogs. American Journal of Veterinary Research, v. 52, n. 4, p. 561-565, 1991.

40.BRIGHT, J. M.; DENTINO, M. Indirect arterial blood pressure measurement in nonsedated Irish Wolfhounds: reference values for the breed. Journal of American Hospital Association, v. 38, n. 6, p. 521-526, 2002.

41.FOX, P. R.; SISSON, D.; MOISE, N. S. Textbook of canine and feline cardiology: principles and clinical practice. 2. ed. Philadelphia: W.B. Saunders, 1999. 955 p. 